\title{
The Burden of Diarrheal Diseases among Children under Five Years of Age in Arba Minch District, Southern Ethiopia, and Associated Risk Factors: A Cross-Sectional Study
}

\author{
Shikur Mohammed and Dessalegn Tamiru \\ Department of Public Health, Arba Minch University, P.O. Box 25121, Arba Minch, Ethiopia \\ Correspondence should be addressed to Shikur Mohammed; m_shikur@yahoo.com \\ and Dessalegn Tamiru; dessalegn@gmail.com
}

Received 8 July 2014; Accepted 1 November 2014; Published 18 November 2014

Academic Editor: Niwat Maneekarn

Copyright (c) 2014 S. Mohammed and D. Tamiru. This is an open access article distributed under the Creative Commons Attribution License, which permits unrestricted use, distribution, and reproduction in any medium, provided the original work is properly cited.

\begin{abstract}
Introduction. In Ethiopia diarrhea is the second cause for clinical presentation among under five-year child population next to pneumonia and it is also more common in rural than in urban areas. Methods. A community based cross-sectional study was conducted in Arba Minch District. Data were collected using structured questionnaire by trained data collectors. To identify predictors of diarrhea the negative binomial regression model was used to predict and control the effect of confounders. Results. The prevalence of diarrhea among under-five children was 30.5\%. This study showed children whose mothers did not attend any formal education were $89 \%$ more likely to develop diarrhea (APR $=1.89$, [95\% CI: 1.35, 2.53]) compared to their counterparts. Similarly, children's being in age category 6-23 months (APR $=2.78$ [95\% CI: 1.72, 4.55]) and mothers' poor hand washing practice $(\mathrm{APR}=2.33$ [95\% CI: 1.80, 4.15]) were found predictors of diarrhea. The study also showed that, out of 180 mothers whose child had got diarrhea, about $31 \%$ of mothers could not give anything to manage the diarrhea. Conclusions. In this study the prevalence of diarrhea was high which was significantly associated with maternal education level, age of the child, and personal hygiene practices. Therefore, women's education level of at least primary school and enhancing community based behavioral change communications using multiple channels (radio) and community health workers are recommended to reduce the occurrence and consequences of childhood diarrhea in the study area.
\end{abstract}

\section{Introduction}

Diarrheal diseases are amongst the most frequent childhood illnesses and leading cause of preventable death, especially among children under five in developing countries. Acute diarrheal diseases are one of the main problems affecting children in the world, reducing their well-being and creating considerable demand for health services [1]. Previous studies showed that diarrheal episode causes the death of 700,000 under-five children. Young children are especially vulnerable to diarrheal disease and the majority of deaths related to diarrhea took place in Africa and South Asia. This is unfortunate since the condition can be easily treated with oral rehydration therapy [1-3].

Diarrhea is defined as a child with loose or watery stool for three or more times during a 24 -hour period $[1,4]$. The frequency and severity of diarrhea are aggravated by lack of access to sufficient clean water and sanitary disposal of human waste, inadequate feeding practices and hand washing, poor housing conditions, and lack of access to adequate and affordable health care [4]. The etiology of diarrhea must be understood to accelerate additional preventive measures. Optimal infant and young child feeding practices could prevent more than 10 percent of deaths from diarrhea and acute respiratory infections $[1,4]$. Better hygiene practices, particularly hand washing with soap and the safe disposal of excreta, could reduce the incidence of diarrhea by 35 percent [2-6].

Studies have shown that, in developing countries like Ethiopia, the occurrence of diarrheal disease among underfive children is complex and the relative contribution of 
each factor varies as a function of interaction between socioeconomic, environmental, and behavioral variables. In many developing countries sociodemographic characteristics like maternal and child age and availability sanitary facilities, hygienic practices, flies infestations, and regular consumption of street food are also some determinant factors for the occurrence of diarrheal disease $[7,8]$. The incidence of diarrhea is higher in the second half of the infant's life when inborn immunity is weak and exposure to contaminated weaning foods increases $[3,9]$.

Suboptimal infant and young child feeding practices are a major contributor to child morbidity and mortality $[1,5$, 10]. The failure to exclusively breastfeed young infants to 6 months and the introduction of liquids and solid foods at the early age of life increase the risk of diarrheal disease and are an important cause of infant and young child morbidity and mortality in Africa. To reduce the diarrheal morbidity and mortality, the clinical and public health practitioner communities must work closely together to identify optimal diagnostic, treatment, and prevention methods. A better understanding of the interaction between persistent diarrhea and malnutrition as causes of mortality has drawn increased attention to the need to expand the scope of intervention programs $[3,5,9,11]$. Oral rehydration solutions (ORS) or commercially available solutions made of appropriate amounts of sodium, potassium, and glucose should be used for rehydration if patients can consume or drink the required volumes; otherwise appropriate intravenous fluids may be used $[10,12]$. Some studies and scholars recommended two general methods for an effective treatment of diarrheal disease, ORS and zinc supplementations [10].

In Ethiopia studies have shown that the prevalence of diarrhea both in urban and in rural areas was $13 \%$. The prevalence of diarrhea was relatively high among children aged from 6 to 23 months. In southern Ethiopia, 25.5\% of children experience diarrhea, but three-fourths occurred among rural children [2]. However, there is no study which documented the magnitude and factors associated with diarrheal disease in the rural communities of Arba Minch Zuria. Therefore, this study aimed to assess the prevalence of diarrhea and associated factors among under-five children in Arba Minch Zuria.

\section{Methods}

This cross-sectional community based study was carried out from January to February (dry season) 2012 in Arba Minch Zuria, Southern Ethiopia. Arba Minch Zuria is one of the 77 woredas (districts) in the Southern Nations, Nationalities and Peoples' Region of Ethiopia. In 2007 Central Statistical Agency reported that this district has 165,680 total populations of which 82,751 were males and 82,929 were females [11]. Most of the people depend on traditional subsistence agriculture. Fruit and vegetables were the two main agricultural products of Arba Minch Zuria [2, 11].

Multistage sampling technique was used to select households. From 29 rural kebeles (small villages), 9 kebeles were randomly selected. Then, 590 mothers of index children aged under five years were selected from reference populations who were obtained from the survey conducted before the main study was commenced. The systematic random sampling technique was used to take the mothers-child pairs from each of the selected kebeles (small villages). In case of more than one child in a given household, the lottery method was used to select single child.

The sample size was calculated using a formula for estimation of a single proportion as follows:

$$
n=\frac{(Z \alpha / 2)^{2} p(1-p)}{d^{2}},
$$

where $Z$ = standard normal variable at $95 \%$ confidence level (1.96); $P=25.5$ (proportion about prevalence of diarhea) [2]; $d=0.05$ (5\% margin of error); and total sample size $=295$.

Considering the design effect, total sample size was multiplied by 2 . So, the final total sample size was 590 . The study included mothers who had children aged less than 5 years and are permanent residents of selected kebeles (small villages). Mothers who come to visit their parents were not included.

Data were collected using WHO core questionnaires [13]. The survey included questions concerning sociodemographics, maternal and child characteristics, child feeding practices, and environmental health conditions. Data collectors were selected from local people depending on their ability to speak the local language and training was given to them. The questionnaire was pretested for its understandability by selecting some subjects before the data collections begin. Based on a pretest additional adjustment was made in terminologies and formatting of the questionnaire. Principal investigators and trained supervisors monitored the overall quality of the data collection.

In this study, diarrhea was defined as a child with loose or watery stool for three or more times during a 24-hour period in the household within two-week period prior to the survey as reported by the mother/caretaker of the child. Prevalence of diarrhea was defined as the total number of diarrhea cases divided by the total number of under-five children in the study in the given time interval.

Water source was defined as improved-water sources if the sources were either of the following: household connections, public standpipes, protected dug wells, and protected springs. Unimproved-water sources included unprotected dug wells, unprotected springs and rivers.

Proper waste disposal was defined as a way of disposing refuses which included burning, burying in a pit or storing in a container, and disposing in the designed site whereas disposing in open fields was considered as an unimproveddisposal method.

Home based water treatment was defined as methods employed for the purposes of treating water in the home using any of the following affordable water treatment techniques like boiling, filtration, and chlorination (chlorine).

Maternal knowledge about diarrhea was defined as knowledge of child caregivers, how diarrhea can be transmitted, and methods controlling diarrheal episodes. Information on maternal knowledge was assessed using a questionnaire consisting of questions on identification of diarrhea, seeking 
medical attention, treatment practices including amount and type of foods given, breastfeeding behavior during illness, prevention of diarrhea, and food handling practices.

Hand washing was defined as if a mother/caretaker had a practice of hand washing after toilet, before child fed, and before having contact with food or any contaminated utensils using clean water and soap or ash which was considered as "good" if not "poor."

The data were entered in double EpiData software, checked for missing values and outliers and analyzed using SPSS (SPSS Inc. version 16.0, Chicago, Illinois). Descriptive statistics were used to describe the study population in relation to relevant variables. To identify independent predictors of childhood diarrhea the negative binomial regression model was used to control the effect of confounders. The test was two-sided and $P<0.05$ was considered statistically significant. We reported the results as an adjusted prevalence ratio (APR) and 95\% confidence intervals.

The study was conducted after getting official permission from an ethical clearance committee of Arba Minch University, College of Medicine and Health Sciences. Informed verbal consent was obtained from each study participant. Each respondent was informed about the objective of the study and privacy during the interview. Participants were informed about ways of prevention and home management of childhood diarrhea after collection of data. ORS was given to the mothers whose child had diarrhea during data collection time with clear instructions. Besides, they were also advised when to take the child to the nearest health facility so as to get professional support.

\section{Results}

A total of 590 households were included in this study and a complete response was obtained from all (100\%) respondents. The mean age of mothers was $29.5(\mathrm{SD} \pm 6.7)$, the range being from 15 to 45 years. The majority of mothers 565 (95.7\%) were married. More than two-thirds (66.90\%) of households had a family size greater than 5 people. The majority of mothers $366(62 \%)$ did not attend formal education (Table 1).

With regard to the sanitary facilities, 540 (91.5\%) of households had latrine and 391 (62.27\%) households got the water from protected spring and piped water. Almost all households 580 (98\%) were living in a house made of mud floor. Majority of dwelling houses 390 (66\%) had no partition room. Fifty (9\%) of the households had no latrine. The mean per capita water consumption of the households was 6.5 (SD \pm 4) litres/day. More than one-third of households (33.7\%) used drinking water from unprotected sources. Only 191 (32.4\%) mothers have a comprehensive knowledge about the cause of diarrhea and ways of transmission (Table 2).

Two-hundred forty-five children (41.5\%) were aged 24 months and above. Among children who were currently breastfeeding, 131 (65.5\%) were exclusively breastfeeding. Findings from this study showed that 180 (30.5\%) children have experienced diarrhea in the last two weeks preceding the survey. The findings of the study also showed that, out of 180 mothers whose child had gotten diarrhea, about $31 \%$ of mothers did not give anything to manage the diarrhea (Table 3).
TABLE 1: Socioeconomic characteristics of the households in Arba Minch Zuria, 2012.

\begin{tabular}{|c|c|c|}
\hline Variables & Frequency & Percent (\%) \\
\hline \multicolumn{3}{|l|}{ Marital status of mothers } \\
\hline Married & 565 & 95.7 \\
\hline Single & 25 & 4.3 \\
\hline \multicolumn{3}{|l|}{ Mothers' educational status } \\
\hline No formal education & 366 & 62.0 \\
\hline Primary school and above & 224 & 38.0 \\
\hline \multicolumn{3}{|l|}{ Occupation of mother } \\
\hline Housewife & 517 & 87.7 \\
\hline Merchant & 41 & 6.9 \\
\hline Others ${ }^{*}$ & 32 & 5.4 \\
\hline \multicolumn{3}{|l|}{ Fathers' educational status } \\
\hline No formal education & 357 & 60.5 \\
\hline Primary and above & 233 & 39.5 \\
\hline \multicolumn{3}{|l|}{ Paternal occupation } \\
\hline Farmers & 517 & 87.60 \\
\hline Merchant & 40 & 6.80 \\
\hline Daily laborer & 15 & 2.50 \\
\hline Others & 18 & 3.05 \\
\hline \multicolumn{3}{|l|}{ Ethnicity } \\
\hline Gamo & 462 & 78.71 \\
\hline Wolayita & 91 & 15.50 \\
\hline Others ${ }^{* *}$ & 34 & 5.79 \\
\hline \multicolumn{3}{|l|}{ Number of U5 } \\
\hline 1 & 395 & 66.9 \\
\hline$>1$ & 195 & 29.1 \\
\hline \multicolumn{3}{|l|}{ Family size } \\
\hline$<5$ & 195 & 29.1 \\
\hline$\geq 5$ & 395 & 66.9 \\
\hline \multicolumn{3}{|l|}{ Maternal age (in years) } \\
\hline$>20$ & 12 & 2.1 \\
\hline $20-24$ & 117 & 19.80 \\
\hline $25-29$ & 186 & 31.5 \\
\hline $30-34$ & 125 & 21.5 \\
\hline$\geq 35$ & 150 & 25.42 \\
\hline \multicolumn{3}{|l|}{ Attended antenatal care } \\
\hline Yes & 446 & 75.6 \\
\hline No & 144 & 24.4 \\
\hline \multicolumn{3}{|l|}{ Religion } \\
\hline Protestant & 371 & 62.9 \\
\hline Orthodox & 205 & 34.75 \\
\hline Muslim & 14 & 2.35 \\
\hline
\end{tabular}

${ }^{*}$ Government employee; daily laborer; ${ }^{* *}$ Amara, Gurage, and Oromo.

Diarrheal disease was significantly associated with maternal education, child age, and maternal hand washing practices. Multiple regression model showed that children whose mothers did not attend any formal education were $89 \%$ more likely to develop diarrhea (APR $=1.89$ [95\% CI: 1.35 , 2.53]) compared to children whose mothers attended formal education (Table 4). 
TABLE 2: The characteristics of children's living environment in Arba Minch Zuria, 2012.

\begin{tabular}{|c|c|c|}
\hline Variables & Frequency $(n)$ & Percent (\%) \\
\hline \multicolumn{3}{|l|}{ Floor of the house } \\
\hline Mud & 439 & 74.4 \\
\hline Cement & 151 & 25.6 \\
\hline \multicolumn{3}{|l|}{ Number of rooms in the house } \\
\hline 1 & 390 & 66 \\
\hline 2 & 110 & 18.6 \\
\hline$\geq 3$ & 90 & 15.4 \\
\hline \multicolumn{3}{|l|}{ Availability of latrine } \\
\hline Yes & 540 & 91.6 \\
\hline No & 50 & 8.4 \\
\hline \multicolumn{3}{|l|}{ Type of latrine } \\
\hline Traditional pit latrine & 438 & 81.1 \\
\hline Ventilated improved latrine & 102 & 18.9 \\
\hline \multicolumn{3}{|l|}{ Waste disposal system } \\
\hline Proper disposal & 475 & 80.5 \\
\hline Open field disposal & 115 & 19.5 \\
\hline \multicolumn{3}{|l|}{ Source of drinking water } \\
\hline Protected source & 391 & 66.3 \\
\hline Unprotected source & 199 & 33.7 \\
\hline \multicolumn{3}{|l|}{ Distance of water source } \\
\hline$\leq 30$ minutes & 503 & 85.4 \\
\hline$>30$ minutes & 87 & 14.6 \\
\hline \multicolumn{3}{|l|}{ Ways of water transportation } \\
\hline Covered container & 496 & 84.1 \\
\hline Uncovered container & 94 & 15.9 \\
\hline \multicolumn{3}{|l|}{ Home based water treatment } \\
\hline Yes & 93 & 15.7 \\
\hline No & 491 & 84.3 \\
\hline \multicolumn{3}{|c|}{ Knowledge about cause of diarrhea } \\
\hline Have knowledge & 191 & 32.4 \\
\hline Have no knowledge & 399 & 67.6 \\
\hline
\end{tabular}

The age of children was also significantly associated with the occurrence of diarrhea. Children aged from 6 to 23 months were about 3 times more likely to develop diarrhea $(\mathrm{APR}=2.78$ [95\% CI: 1.72, 4.55] $)$ compared to children whose age was less than five months.

The risk of developing diarrhea was about 2 times higher among children whose mothers had poor hand washing practice $(\mathrm{APR}=2.33[95 \% \mathrm{CI}: 1.80,4.15])$ compared to children whose mothers had good hand washing practice.

\section{Discussion}

Findings from this study showed that the prevalence of childhood diarrhea among under-five children was about $30.5 \%$, which is relatively high when compared with $13 \%$ reported by Ethiopian Demographic and Health Survey in $2011[2,5]$. That might be due to the inclusion of only rural children and the difference in provision health service between rural and urban population.
TABLE 3: Child demographic and behavioral characteristics in Arba Minch Zuria, 2012.

\begin{tabular}{|c|c|c|}
\hline Variables & Frequency $(n)$ & Percent (\%) \\
\hline \multicolumn{3}{|l|}{ Child sex } \\
\hline Male & 265 & 44.9 \\
\hline Female & 325 & 55.1 \\
\hline \multicolumn{3}{|l|}{ Child's age } \\
\hline 0-5 months & 193 & 32.7 \\
\hline $6-23$ months & 152 & 25.8 \\
\hline 24 months and above & 245 & 41.5 \\
\hline \multicolumn{3}{|l|}{ Birth order } \\
\hline First & 94 & 16.1 \\
\hline Second & 128 & 21.6 \\
\hline Third & 129 & 21.8 \\
\hline Fourth and above & 239 & 40.5 \\
\hline \multicolumn{3}{|l|}{ Exclusive breast feeding } \\
\hline Yes & 131 & 65.5 \\
\hline No & 69 & 34.5 \\
\hline \multicolumn{3}{|l|}{ Measles vaccination } \\
\hline Yes & 56 & 53.3 \\
\hline No & 49 & 46.7 \\
\hline \multicolumn{3}{|l|}{ Hand washing practice } \\
\hline Good & 508 & 86.1 \\
\hline Poor & 82 & 13.9 \\
\hline \multicolumn{3}{|l|}{ Diarrhea } \\
\hline Yes & 180 & 30.5 \\
\hline No & 410 & 69.5 \\
\hline \multicolumn{3}{|c|}{ Home management of diarrhea } \\
\hline Yes & 56 & 31.11 \\
\hline No & 124 & 68.89 \\
\hline \multicolumn{3}{|l|}{ Child feeding methods } \\
\hline Cup & 155 & 26.30 \\
\hline Spoon & 100 & 16.95 \\
\hline Hand & 180 & 30.51 \\
\hline Bottle & 25 & 4.24 \\
\hline Not any instruments & 130 & 22 \\
\hline
\end{tabular}

This study indicated that maternal education status had a significant contribution to preventing diarrheal morbidity. Children whose mothers did not attend formal education were more likely to develop diarrhea compared to children whose mothers did not attend any formal education. Findings from Ghana and Nigeria also showed that the prevalence of diarrhea varies according to education status of children's mothers, which is relatively high among children whose mothers had no education $[8,14,15]$. This might be due to health education given on hygiene, feeding and weaning practices and the management of childhood illness at school, and the role of different school clubs in personal hygiene and environmental sanitation.

This study showed that availability of latrine, types of floor, waste disposal system, and the source of water were not significantly associated with diarrheal morbidity an inverse of studies from Ghana, where availability of water, sanitary 
TABLE 4: Factors associated with diarrheal disease among under-five children in Arba Minch Zuria District, Southern Ethiopia, 2012.

\begin{tabular}{|c|c|c|c|c|}
\hline \multirow{2}{*}{ Predictors } & \multicolumn{2}{|c|}{ Diarrhea } & \multirow{2}{*}{ CPR $(95 \%$ CI $)$} & \multirow{2}{*}{$\operatorname{APR}(95 \% \mathrm{CI})$} \\
\hline & Yes & No & & \\
\hline \multicolumn{5}{|l|}{ Mother's education } \\
\hline No formal education & $125(34.2 \%)$ & $241(65.8 \%)$ & $1.39(1.06,1.82)^{*}$ & $1.89(1.35,2.53)^{*}$ \\
\hline Primary school and above & $55(24.6 \%)$ & $169(75.4 \%)$ & 1.00 & 1.00 \\
\hline \multicolumn{5}{|l|}{ Floor of the house } \\
\hline Mud & $145(33 \%)$ & $294(67 \%)$ & $1.42(1.04,1.96)^{*}$ & $1.22(0.73,2.04)$ \\
\hline Cement & $35(23.2 \%)$ & $116(76.8 \%)$ & 1.00 & 1.00 \\
\hline \multicolumn{5}{|l|}{ Knowledge about diarrhea } \\
\hline Had knowledge & $110(27.6 \%)$ & $289(72.4 \%)$ & $0.75(0.59,0.96)^{*}$ & $0.71(0.45,1.12)$ \\
\hline Had no knowledge & $70(36.6 \%)$ & $121(63.4 \%)$ & 1.00 & 1.00 \\
\hline \multicolumn{5}{|l|}{ Latrine available } \\
\hline No & $15(30 \%)$ & $35(70 \%)$ & $0.97(0.63,1.53)$ & \multirow{2}{*}{-} \\
\hline Yes & $165(30.5 \%)$ & $375(69.5 \%)$ & 1.00 & \\
\hline \multicolumn{5}{|l|}{ Waste disposal system } \\
\hline Improper disposal & $39(33.9 \%)$ & $76(66.1 \%)$ & $1.14(0.85,1.53)$ & \multirow{2}{*}{ - } \\
\hline Proper disposal & $141(29.7)$ & $334(70.3)$ & 1.00 & \\
\hline \multicolumn{5}{|l|}{ Source of drinking water } \\
\hline Unprotected source & $66(33.2 \%)$ & $133(66.8 \%)$ & $1.14(1.05,1.49)^{*}$ & $1.32(0.97,1.72)$ \\
\hline Protected source & $114(29.2)$ & $277(70.8 \%)$ & 1.00 & 1.00 \\
\hline \multicolumn{5}{|l|}{ Age of the child } \\
\hline 0-5 months & $45(23.3 \%)$ & $148(76.7 \%)$ & 1.00 & 1.00 \\
\hline 6-23 months & $60(39.5 \%)$ & $92(60.5 \%)$ & $1.69(1.23,3.34)^{*}$ & $2.78(1.72,4.55)^{*}$ \\
\hline 24 months and above & $75(30.6 \%)$ & $170(69.4 \%)$ & $1.31(0.96,1.80)$ & $1.52(0.86,2.70)$ \\
\hline \multicolumn{5}{|l|}{ Hand washing practice } \\
\hline Poor & $44(54.4 \%)$ & $38(45.6 \%)$ & $1.83(1.44,2.33)^{*}$ & $2.33(1.80,4.15)^{*}$ \\
\hline Good & $149(28.8 \%)$ & $359(71.2 \%)$ & 1.00 & 1.00 \\
\hline
\end{tabular}

*Statistical significance at $P<0.05$, CPR: crude prevalence ratio, APR: adjusted prevalence ratio.

facilities, and hygienic practices were positively associated with the occurrence of diarrheal disease $[2,8]$. This might be due to differences in culture of waste management and seasonal variation.

The children aged from 6 to 23 months were at high risk of developing diarrhea compared to children aged less than five months. The findings from Thailand [16] also showed that children whose ages from 6 to 23 months were more endanger of diarrheal disease than other age groups. This might be due to transition from an exclusive breastfeeding to introduction of complementary food and poor maternal hand washing and an increased introduction of solid foods which is unsafe and poor in hygiene to children whose immunity was not well developed.

This study also indicated that poor maternal hand washing practices were positively associated with the occurrence of diarrheal morbidity. This indicates that since the mothers were the main caregivers for their children they should wash their hands before feeding infants and young children to tackle the occurrence of hygiene related disease. Studies have also indicated that the proper hand washing before feeding this vulnerable age group has great role in the prevention of diarrhea and other diseases $[2,15,17]$.
A large number of mothers, whose child had diarrhea, did not do anything to manage the diarrhea at home level. For the better management of diarrhea the national guideline recommends counseling mothers on the three rules of home treatments: give extra fluid, continue feeding, and advise the mother when to return to health facility [18]. Therefore, the health planners and health practitioners should work hard to bring behavioral change among the community for better perfection and management of diarrhea.

The findings of this study can be used as baseline data for policy makers in formulation, national infants and young health guideline and achievement of millennium development goals in the reduction of child mortality. Using WHO core questionnaire and community based study design is the strength of this study. However, the cross-sectional nature of this study limited drawing causal inferences from the association between the factors and the occurrence of diarrhea. There might be also reporting bias during face to face interviews which is tried to be minimized by selecting data collectors from the native local language speakers. There might be also recall bias, but we tried to limit recall bias by checking the card and the records about the child by health extension workers. Since this study was collected during dry 
season it might be difficult to consider the impacts of seasonal variation.

\section{Conclusions}

The findings of this study showed that the prevalence of diarrhea is high $(30.5 \%)$. The occurrence of diarrhea was positively associated with maternal education, age of the child, and personal hygiene. Therefore, the findings in this study have important policy implications for health intervention and support the view that investing in girls' education may have substantial benefits for child health. Women's education level of at least primary should be achieved to reduce childhood diarrhea. Reducing diarrhea involves providing better sanitation for the entire population and the hygiene of the person caring for the child through community health workers. Although prevention is better than cure, sometimes prevention activities might fall and lead to morbidity. Therefore, counseling mothers on the three rules of home treatment (give extra fluid, continue feeding, and advise the mother when to return to health facility) is very crucial for the control and the prevention of the disease. We recommend further study to identify factors affecting home management of childhood diarrhea.

\section{Conflict of Interests}

The authors declare that there is no conflict of interests regarding the publication of this paper.

\section{Authors' Contribution}

Shikur Mohammed (the corresponding author) designed and supervised the study and ensured the quality of the data and worked on the analysis and interpretation of the data. Dessalegn Tamiru participated in the design and supervision of the study and reviewed the paper for publication. The authors read and approved the final paper.

\section{Acknowledgments}

The authors would like to express their sincere gratitude to the Arba Minch University, College of Medicine and Health Sciences for facilitating the study. They would also like to acknowledge the study participants for their diligence and dedication in collecting and inputting high quality data.

\section{References}

[1] World Health Organization (WHO), Integrated Management of Childhood Illness, 2010.

[2] Central Statistical Authority and ORC Macro, Ethiopia Demographic and Health Survey 2011, Addis Ababa, Ethiopia, 2011.

[3] C. L. Fischer Walker, I. Rudan, L. Liu et al., "Global burden of childhood pneumonia and diarrhoea," The Lancet, vol. 381, no. 9875, pp. 1405-1416, 2013.

[4] T. Gerald, O. Keusch, and B. Alok, Disease Control Priorities in Developing Countries, World Bank, Washington, DC, USA, 2001.
[5] Federal Ministry of Health, National Strategy for Child Survival in Ethiopia, Family Health Department, Addis Ababa, Ethiopia, 2005.

[6] World Health Organization, WHO World Water Day Report, WHO, Geneva, Switzerland, 2000.

[7] K. Yassin, "Morbidity and risk factors and diarrheal diseases among under-five children in rural Upper Egypt," Journal of Tropical Pediatrics, vol. 46, no. 5, pp. 282-287, 2000.

[8] K. Oadi and M. Kuitunen, "Childhood diarrheal morbidity in the Accra Metropolitan Area, Ghana: socio-economic, environmental and behavioral risk determinants," Journal of Health and Population in Developing Countries, Article ID 17646, pp. 33-46, 2005.

[9] A. G. Yohannes, K. Streatfield, and L. Bost, "Child morbidity patterns in Ethiopia," Journal of Biosocial Science, vol. 24, no. 2, pp. 143-155, 1992.

[10] USAID, Guidelines for New Diarrhea Treatment Protocols for Community-Based Healthcare Workers, 2005.

[11] Central Statistical Agency, "Summary of statistical report of 2007," Population and Housing Census, Central Statistical Agency, Addis Ababa, Ethiopia, 2007.

[12] Center for Disease Control, Guidelines for the Management of Acute Diarrhea, Department of Health and Human Services in United States, 2008.

[13] WHO/UNICEF, Core Questions on Drinking Water and Sanitation for Household Surveys, WHO Press, Geneva, Switzerland, 2006.

[14] C. S. Yilgwan and S. N. Okolo, "Prevalence of diarrhea disease and risk factors in Jos University Teaching Hospital, Nigeria," Annals of African Medicine, vol. 11, no. 4, pp. 217-221, 2012.

[15] C. S. Yilgwan, G. Yilgwan, and I. I. Abok, "Domestic water sourcing and the risk of diarrhea: a cross-sectional survey of a semi-urban community in Nigeria," Journal of Medicine, vol. 5, no. 1, pp. 34-37, 2005.

[16] W. Calistus and P. Alessio, "Factors associated with diarrhea among children less than 5 years old in Thailand: a secondary analysis of Thailand multiple indicator cluster survey," Journal of Health Research, vol. 23, pp. 17-22, 2009.

[17] G. O. Vieira, L. R. Silva, and T. D. O. Vieira, "Child feeding and diarrhea morbidity," Jornal de Pediatria, vol. 79, no. 5, pp. 449454, 2003.

[18] Drug Administration and Control Authority of Ethiopia Contents, Standard Treatment Guideline for Primary Hospitals, 2010. 


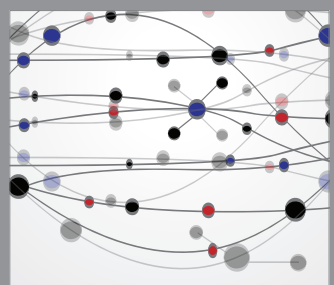

The Scientific World Journal
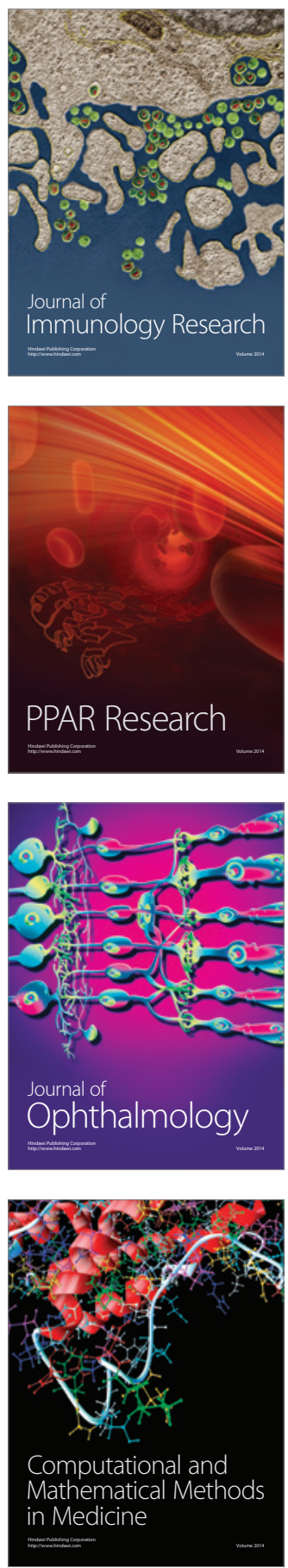

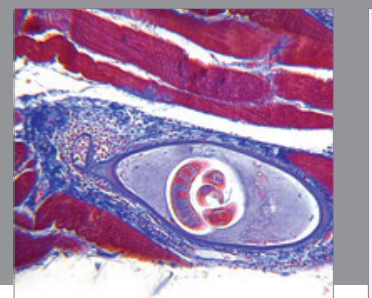

Gastroenterology

Research and Practice
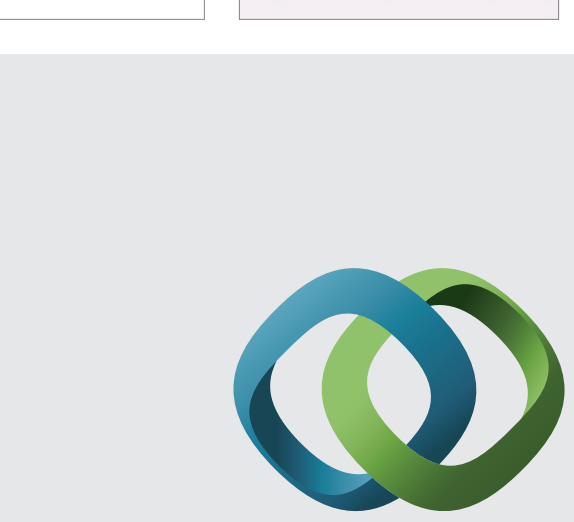

\section{Hindawi}

Submit your manuscripts at

http://www.hindawi.com
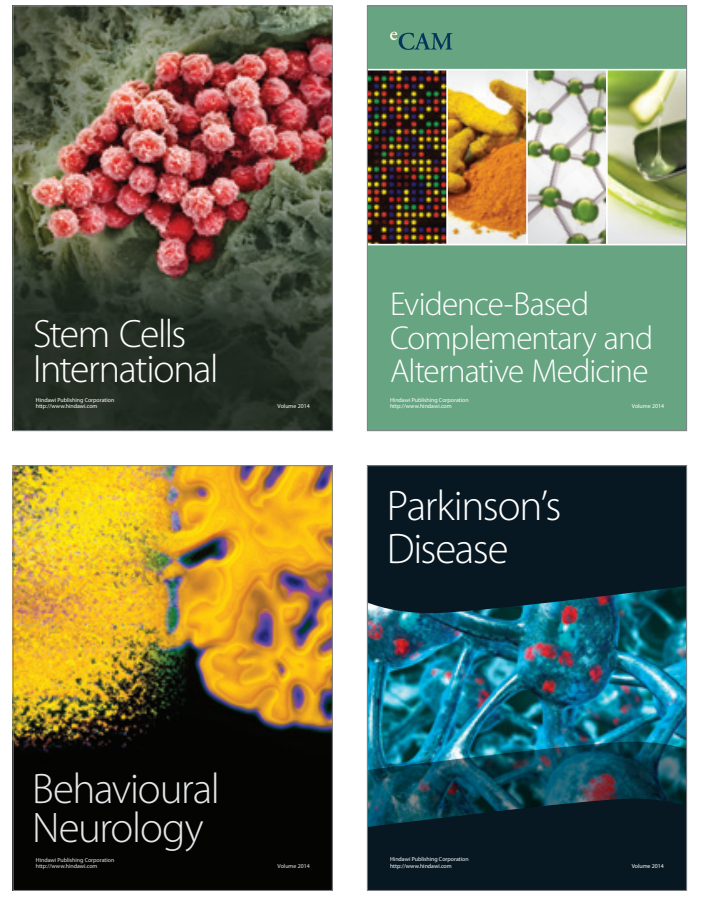
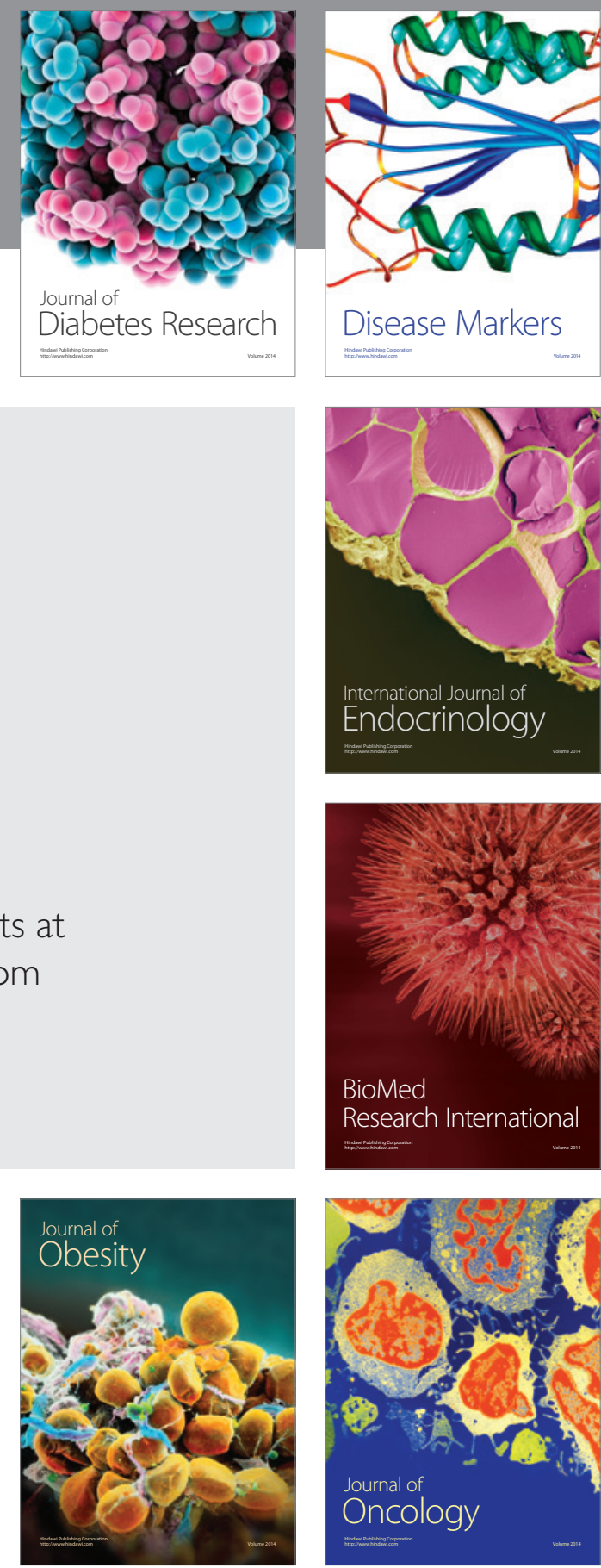

Disease Markers
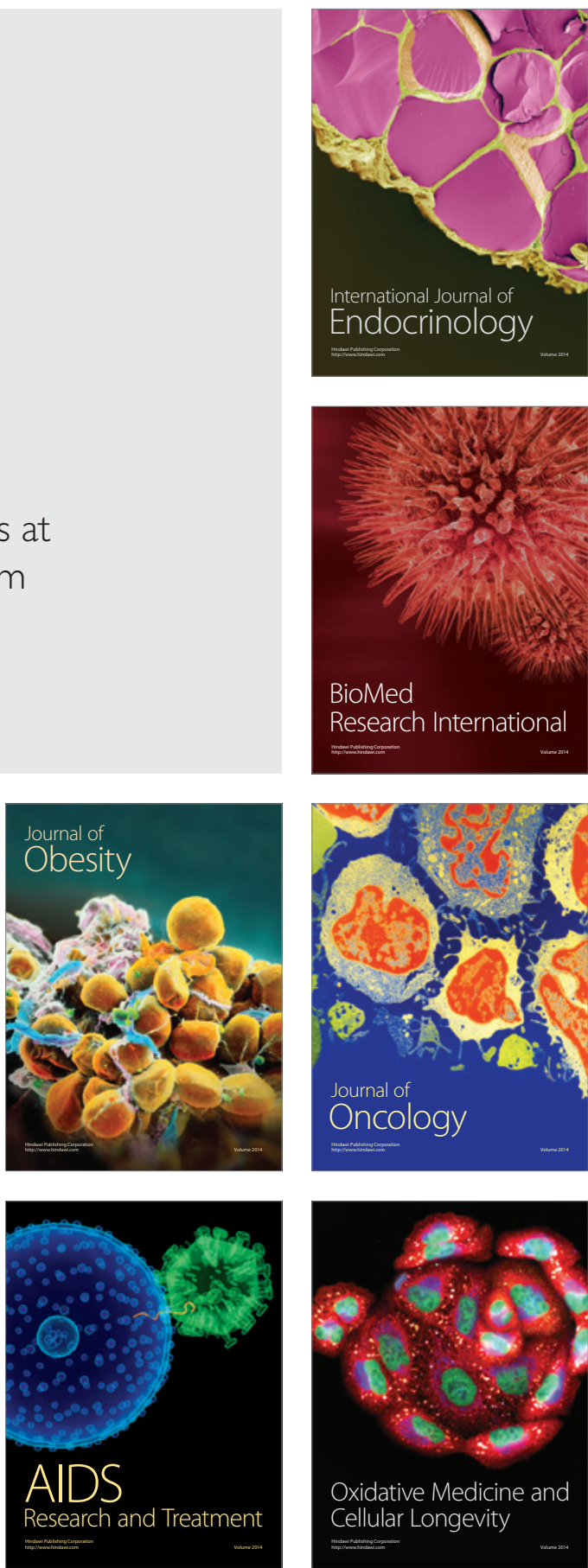